\title{
Inclusive Searches for Supersymmetry with Jets and Missing Transverse Energy with the ATLAS Detector
}

\author{
Marija Vranjes Milosavljevic ${ }^{* \dagger}$ \\ Institute of Physics Belgrade \\ E-mail: marija.vranjes.milosavljevic@cern.ch
}

\begin{abstract}
This document summarizes a first set of measurements of most important kinematical variables for SUSY searches in the final states with jets and missing transverse momentum from the $\sqrt{s}=$ $7 \mathrm{TeV}$ proton-proton collisions at the LHC. The analyzed data were collected during the period March to July 2010 with the ATLAS detector and correspond to a total integrated luminosity of $70 \pm 8 \mathrm{nb}^{-1}$. Good agreement between data and Monte Carlo simulations has been found which implies that Standard Model backgrounds in these searches are under control.
\end{abstract}

35th International Conference of High Energy Physics

July 22-28, 2010

Paris, France

* Speaker.

$\dagger$ on behalf of ATLAS Collaboration 


\section{Introduction}

Supersymmetry (SUSY) [1] is one of the best motivated theories to extend the Standard Model in the search for a more comprehensive framework.

This document describes a first comparison of data to Monte Carlo simulations for kinematical variables sensitive to a presence of SUSY particles in searches involving jets and missing transverse momentum with no leptons. Selections based on these variables are expected to be sensitive not only to R-parity conserving SUSY models, but also to any model in which strongly interacting particles give final states containing jets and missing momentum. The measurements reported here are based on the $\sqrt{s}=7 \mathrm{TeV}$ LHC proton-proton collisions that were recorded between March and July 2010 with the ATLAS detector and correspond to a total integrated luminosity of $70 \pm 8 \mathrm{nb}^{-1}$.

\section{Event selection}

After rejection of events which have at least one jet not associated to collisions, such as sporadic noise in the hadronic endcap and electromagnetic calorimeters or cosmic ray energy deposits [2], events with no isolated leptons are categorized in four classes according to final selection requirements on jet momenta, the transverse missing energy $E_{\mathrm{T}}^{\text {miss }}$ measured by calorimeter cells belonging to topological clusters at the electromagnetic scale, the effective mass $M_{\text {eff }}$ defined as scalar sum of transverse momenta of two to four jets defining the analysis channel and $E_{\mathrm{T}}^{\text {miss }}$, and the angle between the jets and the $E_{\mathrm{T}}^{\text {miss }}$ two-vector, $\Delta \phi$ (jet, $\left.E_{\mathrm{T}}^{\text {miss }}\right)$, as described in Table 1.

Table 1: Cuts on the $p_{\mathrm{T}}$ of the leading jet, the $p_{\mathrm{T}}$ of the other jets and the missing transverse momentum used for each of the studied jet multiplicities.

\begin{tabular}{|l|c|c|c|c|}
\hline Number of jets & Monojets & $\geq 2$ jets & $\geq 3$ jets & $\geq 4$ jets \\
\hline Leading jet $p_{\mathrm{T}}(\mathrm{GeV})$ & $>70$ & $>70$ & $>70$ & $>70$ \\
Subsequent jets $p_{\mathrm{T}}(\mathrm{GeV})$ & veto if $>30$ & $>30$ & $>30($ Jets 2 and 3) & $>30$ (Jets 2 to 4) \\
\hline$E_{\mathrm{T}}^{\text {miss }}(\mathrm{GeV})$ & $>40$ & $>40$ & $>40$ & $>40$ \\
$\Delta \phi\left(\right.$ jet $\left._{i}, E_{\mathrm{T}}^{\text {miss }}\right)(\mathrm{rad})$ & no cut & {$[>0.2,>0.2]$} & {$[>0.2,>0.2,>0.2]$} & {$[>0.2,>0.2,>0.2,>0]$} \\
\hline$E_{\mathrm{T}}^{\text {miss }}>f \times M_{\mathrm{eff}}$ & no cut & $f=0.3$ & $f=0.25$ & $f=0.2$ \\
\hline
\end{tabular}

\section{Results}

Distributions of most important kinematical variables in channels defined by jet multiplicities and selections described in Table 1 are presented in Figure 1. The total uncertainty shown as yellow band in Figure 1(a-f) is dominated by the uncertainties on the jet energy scale, normalization factors for the Monte Carlo cross sections and the luminosity. Details on Monte Carlo samples used in this study and their cross sections can be found in [3]. Figure 1(a) shows the missing transverse momentum distribution for events in the monojet channel before any $E_{\mathrm{T}}^{\text {miss }}$ requirement is made. The difference in azimuthal angle between the jet and the missing transverse momentum vector in monojet events selected with $E_{\mathrm{T}}^{\text {miss }}>40 \mathrm{GeV}$ cut is shown in Figure 1(b). The distributions of stransverse, $m_{\mathrm{T} 2}$ and contransverse mass, $m_{\mathrm{CT}}$ [3] for 2-jet selection are shown in Figure 1(c) and 1 (d) after requiring missing transverse momentum greater than $40 \mathrm{GeV}$. For both distributions SUSY signal would be expected to be present at high values of these variables. Distributions of effective mass after the $E_{\mathrm{T}}^{\text {miss }}$ cut are shown for three-jet and four-jet events in Figure 1(e) and 1(f). All distributions are reasonably well described by the Monte Carlo predictions, given the 
systematic uncertainties. For illustrative purposes all presented distributions are compared to the prediction from SU4 supersymmetric mSUGRA benchmark point with $m_{0}=200 \mathrm{GeV}, m_{1 / 2}=160$ $\mathrm{GeV}, A_{0}=-400 \mathrm{GeV}, \tan \beta=10$ and $\mu>0$. More distributions of supersymmetry-sensitive variables can be found in [3].

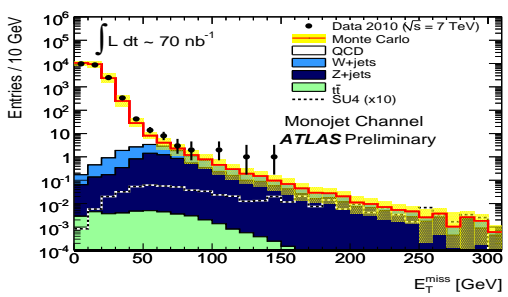

(a)

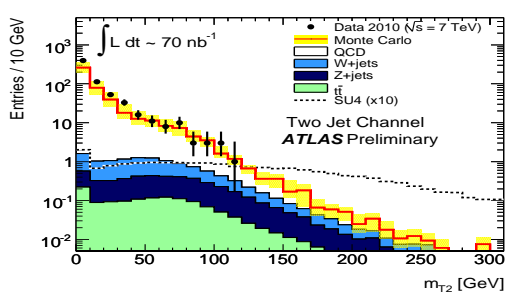

(c)

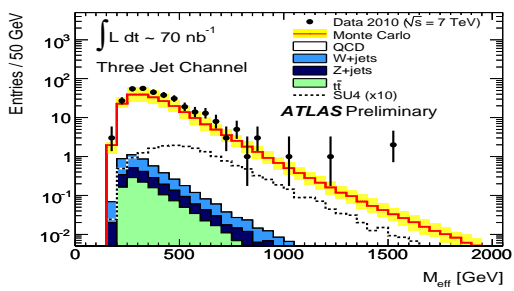

(e)

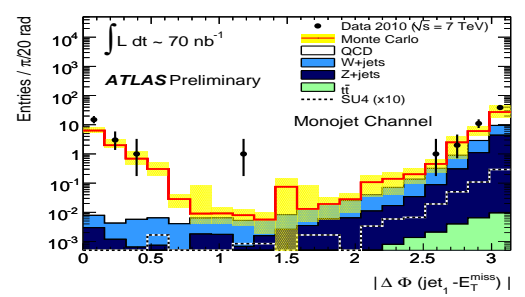

(b)

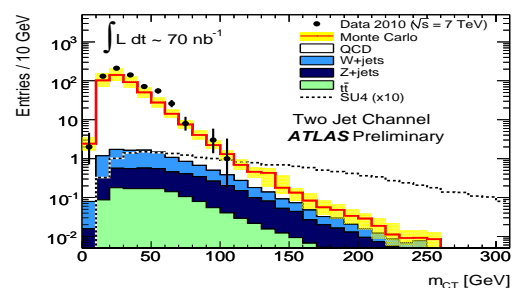

(d)

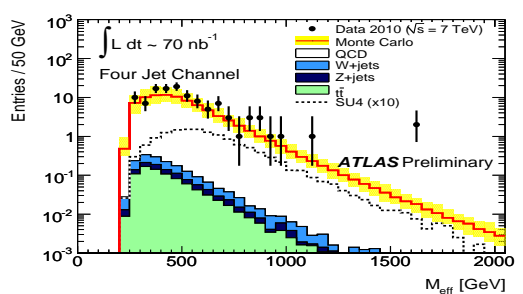

(f)

Figure 1: Distributions of the missing transverse momentum (a) and difference in azimuthal angle between the jet and the missing transverse momentum vector (b) for events in the monojet channel, stransverse mass $m_{\mathrm{T} 2}$ (c) and the contransverse mass $m_{\mathrm{CT}}$ (d) for events in the dijet channel, effective mass for events in the three-jet channel (e) and four-jet channel (f). The cut requiring $E_{\mathrm{T}}^{\text {miss }}>40 \mathrm{GeV}$ has been applied on (b), (c), (d), (e), (f).

\section{Summary}

An analysis of the first $70 \pm 8 \mathrm{nb}^{-1}$ of integrated luminosity collected with the ATLAS detector is presented. Measured distributions of missing transverse momentum, effective mass, azimuthal angles, stransverse mass and contransverse mass show agreement with the Standard Model expectations within the systematic uncertainties achievable so far.

\section{References}

[1] Y.A. Golfand and E.P. Likhtman, JETP Lett. 13 (1971) 323-326; A. Neveu and J.H. Schwarz, Nucl. Phys. B31 (1971) 86-112; A. Neveu and J.H. Schwarz, Phys. Rev. D4 (1971) 1109-1111.

[2] The ATLAS collaboration, ATLAS-CONF-2010-038, http://cdsweb.cern.ch/record/1277678.

[3] The ATLAS Collaboration, ATLAS-CONF-2010-065, http://cdsweb.cern.ch/record/1281340 . 